\title{
Clinical Event Pattern
}

National Cancer Institute

\section{Source}

National Cancer Institute. Clinical Event Pattern. NCI Thesaurus. Code C105760.

The pattern of a clinical event. 